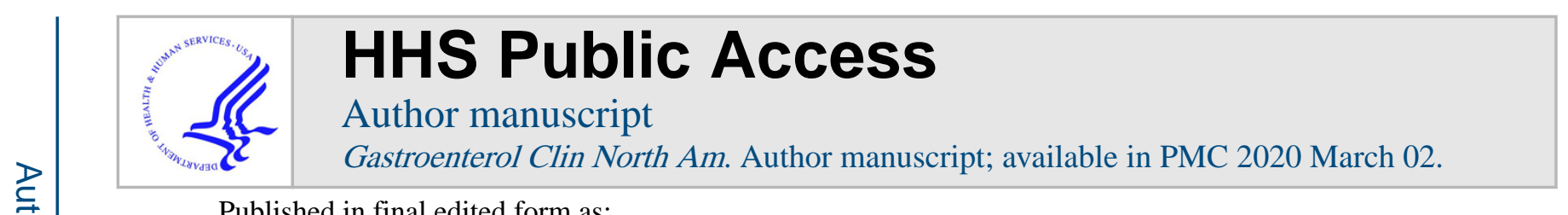

Published in final edited form as:

Gastroenterol Clin North Am. 2018 December ; 47(4): xv-xvi. doi:10.1016/j.gtc.2018.07.017.

\title{
Pediatric Gastroenterology
}

\author{
Robert J. Shulman, MD \\ Baylor College of Medicine, Children's Nutrition Research Center, Texas Children's Hospital, 1100 \\ Bates Street, Houston, TX 77030, USA
}

Robert J. Shulman, MD

It is an honor and a privilege to have helped bring this issue of Gastroenterology Clinics of North America to you. Pediatric gastroenterology continues to grow as a vibrant field, helping to improve the health of children through advances in clinical medicine and research. This issue reflects both of these areas. Ever-improving molecular techniques are helping to define new disorders and clarify existing ones, and pointing the way to new therapies; these advances are reflected in the topics presented here.

The articles are written by experts in their respective fields who have extensive experience in clinical management of the diseases/disorders described and are also key researcherspushing the boundaries of our understanding of the pathogenesis of these ailments.

Importantly, they are also excellent writers, and I believe you will find the articles engaging and clearly written. It certainly was a great learning experience for me, and I believe it will be for you as well.

I am greatly indebted to the authors for their willingness to contribute their time and expertise not only to this issue of Gastroenterology Clinics of North America but also to the field of pediatric gastroenterology. My hope is that they not only will impart new knowledge to us but also inspire others to join in the pursuit of expanding that knowledge so that all of us can do better by our patients.

The topics cover the gamut of problems commonly encountered in pediatric gastroenterology practice. Each article outlines the clinical presentation and most up-to-date management and treatment strategies. In addition, our current understanding of the cause of each disorder is outlined with implications for future treatments. Controversies in management, when present, also are discussed.

The articles are a mirror of pediatric gastroenterology in the early twenty-first century and reflect how far we have come and where we need to go as we strive to move the field of pediatric medicine forward, while maintaining what makes medicine special: the touch of empathy from practitioner to patient, in the face of forces that, at times, make that more and more difficult.

rshulman@bcm.edu. 


\section{Acknowledgments}

Finally, I want also to thank Dr Alan Buchman for the invitation to edit this issue, and Kerry Holland and Sara Watkins at Elsevier for their efforts in bringing this issue to fruition.

\section{Biography}

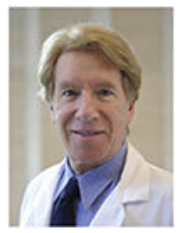

